\title{
The Full Surplus Extraction Theorem with Hidden Actions
}

\author{
Ichiro Obara ${ }^{12}$ \\ Department of Economics \\ University of Minnesota
}

January 19, 2008

\footnotetext{
${ }^{1}$ E-mail: obara@umn.edu

${ }^{2}$ I am grateful to Jacques Crémer, Paul Milgrom, and especially to Tomas Sjöström for their helpful comments. I would also like to thank the seminar audience at many places, inluding NBER/NSF Decentralization Conference 2003 at Purdue, SWET at UC Irvine, Arizona State, Essex, LSE, MEDS, Minnesota, Penn State, Rutger, Stanford, UC Riverside, and UT Austin.
} 


\begin{abstract}
Consider a situation in which a principal commits to a mechanism first and then agents choose unobservable actions before their payoff-relevant types are realized. The agents' actions may affect not only their payoffs directly but also the distribution of their types as well. This paper extends Crémer and McLean's full surplus extraction theorem to such a setting. In this environment, it is shown that a principal may not succeed in extracting full surplus from agents when there are many actions to which the agents can deviate.

However, it is also shown that a principal can extract full surplus generically given any approximately efficient (completely) mixed action profile. This is achieved by using a general mechanism where agents announce both their types and their realized actions. Therefore, with hidden actions, there is a big gap between exact full surplus extraction and approximate full surplus extraction.

JEL classification: C72, D44, D82

Keywords: auction, information acquisition, full surplus extraction, hidden action, mechanism design, mixed strategy, moral hazard, private strategy, virtual implementation.
\end{abstract}




\section{Introduction}

The theory of mechanism design provides a powerful tool to analyze a variety of situations of which asymmetric information is an important aspect. One of the standard assumptions behind this theory is that distributions of private characteristics or types are exogenous. However, this assumption is not always the most reasonable one. There are many situations where the distribution of private types is endogenous rather than exogenous. For example, consider a procurement auction where the potential bidders invest their resources before deciding whether to participate in the auction or not. The bidders may make such investments for a variety of reasons. They may be uncertain about the cost of the project, thus try to come up with a better estimate of it. Or they may spend money for $\mathrm{R} \& \mathrm{D}$ to reduce their costs. In either case, the distribution of private types is endogenous.

Then questions arise naturally; do the standard results with exogenous types generalize to the case with endogenous types? If not, how do they need to be modified? The goal of this paper is to understand when full surplus extaction (FSE hereafter) becomes more difficult (or easier) to obtain with endogenous types.

Crémer and McLean [13](CM hereafter) is the seminal paper on FSE. It identifies the necessary and sufficient condition for FSE for the standard private value auction. Roughly saying, an auctioneer can extract full surplus from bidders when their types are "correlated". This condition is so weak that it is satisfied for almost all information structure with finite type spaces. ${ }^{1}$ The independent value case, which is most popular in the applied literature, is a "rare" exception where FSE is not obtained. This result was later extended by McAfee and Reny [28] to the case of continuous type spaces. ${ }^{2}$

These results, if taken literally, call into the question the robustness of the standard model with risk neutral agents and independent types. But there are two issues here. First, it is hard to believe that no surplus is left to any agent. Second, we need a very sophisticated mechanism for FSE, but we hardly find such a mechanism in real world. Thus there is a sense in which something is missing from the standard model, which prevents a principal from extracting full surplus. ${ }^{3}$ This paper does not address the second (very important) issue (why are real mechanisms so simple?), but addresses the first issue (why may

\footnotetext{
${ }^{1}$ This is not true with infinite type spaces. See Heifetz and Neeman [18].

${ }^{2} \mathrm{~A}$ recent paper by Kosenok and Severinov [21] proves that FSE is possible even with an informed principal.

${ }^{3}$ Crémer and McLean [13] suggests that the common prior assumption may be a cause for their result.
} 
agents secure informational rents?).

My first main result shows that one possible reason for the failure of FSE is the endogeneity of type distributions. Notice that the definition of FSE needs to be modified with endogenous type distributions. For FSE, I require not only that the efficient allocation be implemented, but also that the efficient action profile be implemented. Then the agents may have incentive to choose a less efficient action to save cost or manipulate the type distributions to their advantage. Since the principal needs to take into account these additional constraints, she is severely constrained when the agents can manipulate their type distribution in many ways. Thus, intuitively, FSE becomes more difficult to obtain with more actions. I show exactly how much FSE becomes more difficult with hidden actions by deriving the necessary and sufficient condition for FSE (Theorem 4) in Section 3. One important implication of the result is that FSE may not be a generic property when there are many actions compared to the number of types (Theorem 5).

However, it is also shown that CM's FSE theorem is far more robust to the introduction of hidden actions than it appears to be. Only the implementation of pure action profiles is considered until Section 4 because the efficient action profile is typically unique. In Section 4, I consider the implementation of almost efficient mixed action profiles and show that approximate full surplus extraction is generically possible. Furthermore, the sufficient condition for approximate FSE is much weaker than the condition for exact full surplus extraction (Theorem 7). In particular, it is satisfied generically for symmetric models, contrary to the case with exact FSE. (Theorem 8).

To understand this result, it is useful to extend an agent's type space from $S_{i}$ (type) to $S_{i} \times A_{i}$ (type $\times$ action). Let's call this latter space player $i$ 's extended type space. When implementing a pure action profile, it is without loss of generality to use a mechanism in which the agents announce their types only (Proposition 1 proves this formally). When mixed actions are played, however, the agents have additional private information, namely, a realization of their mixed actions. Thus one is naturally led to a more general mechanism in which the agents announce their extended types. The almost full surplus extraction result hinges on the fact that CM's condition for FSE holds generically for the extended type space $\prod_{i} S_{i} \times A_{i}$. Then, for almost all information structure, the principal can extract full surplus conditional on every $(s, a)$ given any completely mixed action profile. This in turn guarantees that the agents indeed have incentive to play any mixed action profile. Since the efficient action profile can be approximated arbitrarily closely by a completely mixed action profile, the principal can implement an almost efficient mixed action profile (Abreu and Sen [1], Matsushima [26]) and extract the full surplus. 
This paper is hardly the first attempt to consider endogenous types in mechanism design. Probably the most famous class of models with endogenous types would be the model of information acquisition. There are many papers about information acquisition in a variety of contexts ${ }^{4}$ But there are many other examples. Team production is one example. ${ }^{5}$. Another example is a procurement auction with $R \& D$ investment, where the level of $R \& D$ investment at the pre-auction stage determines the distribution of costs. ${ }^{6}$

A few papers offer an explanation as to why FSE may fail. Heifetz and Neeman [18], Neeman [32], and Parreiras [35] present a certain kind of type spaces where FSE cannot be obtained. These papers are discussed in the end of Section 3. Robert [38] introduces risk aversion/limited liability. Laffont and Martimort [22] introduce collusion proofness as an additional constraint on the design of mechanisms for public good problems. ${ }^{7}$

The basic model and some preliminary results are presented in the next section. In Section 3, I extend CM's FSE theorem to the case with endogenous types and show that FSE may not be generic. Section 4 proves the almost full surplus extraction theorem and shows that it is a generic result.

\section{The Model and Some Preliminary Results}

\subsection{The Model}

Consider a principal and $n$ agents. The game proceeds as follows. First the principal proposes a mechanism to the agents, which consists of message spaces, an allocation rule, and monetary transfers. Then the agents choose unobservable actions simultaneously and observe their private types. ${ }^{8}$ The distribution of types depends on the agents' actions. Given their types, the

\footnotetext{
${ }^{4}$ See Milgrom [30] and subsequent papers on information acquisition in auction, which include Bergemann and Välimäki [6], Matthews [27], Persico [35], and Tan [39]. See Crémer and Khalil [10], Crémer, Khalil and Rochet [12] and Lewis and Sappington [23] for information acquision in contracting problems. For recent works on information acquisition and committe decision, see Cai [8], Gerardi and Yariv [16], Gershkov and Szentes [17], Martinelli [27], and Persico [36].

${ }^{5}$ For example, see Sjöström [40].

${ }^{6}$ See Arozamena and Cantillon [3], Tan [39], and Piccione and Tan [37].

${ }^{7}$ Che and Kim [10] shows that collusion proofness is not binding with more than two agents.

${ }^{8}$ This "action" can be a complex strategy. For example, suppose that an agent takes a sequence of actions, each of which depends on private information generated by the preceding actions. Then his action corresponds to a complete contingent strategy and his type corresponds to a sequence of private information.
} 


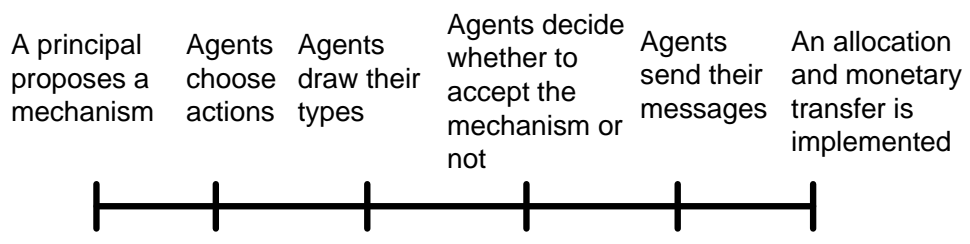

Figure 1:

agents decide whether to participate in the mechanism or not. For the sake of simplicity, it is assumed that agents do not observe who participates in the mechanism. ${ }^{9}$ If they accept the mechanism, they send a message to the principal, and an allocation and monetary transfers are implemented as a function of messages according to the proposed mechanism. The time line of the game is shown in Figure 1. ${ }^{10}$

Let's introduce formal notations. The set of agents is $N=\{1, \ldots, n\}$. Agent $i^{\prime}$ s action set and type space is a finite set $A_{i}$ and $S_{i}$ respectively. It is assumed that $\left|S_{i}\right| \geq 2$ for all $i \in N$. The distribution on $S=\prod_{i=1}^{n} S_{i}$ given $a \in A=$ $\prod_{i=1}^{n} A_{i}$ is given by $\pi: S \times A \rightarrow \Re_{+}$that satisfies $\sum_{s \in S} \pi(s \mid a)=1$ for all $a \in A$. Let $\Xi$ be the space of all such $\pi$. A triple $(\pi, S, A)$ is information structure. Let $\pi_{i}\left(s_{i} \mid a\right)=\sum_{s_{-i} \in S_{i}} \pi\left(s_{i}, s_{-i} \mid a\right)$ be the marginal distribution on $S_{i}$ given $a$. It is assumed that $\pi_{i}\left(s_{i} \mid a\right)>0$ for all $a \in A$ and $s_{i} \in S_{i}$. Let $\pi_{i}\left(s_{-i} \mid s_{i}, a\right)=$ $\frac{\pi(s \mid a)}{\pi_{i}\left(s_{i} \mid a\right)}$ be agent $i^{\prime}$ s subjective distribution over the other agents' types given his type $s_{i}$ and $a$. A $\left|S_{-i}\right|$-dimensional vector $\left(\pi_{i}\left(s_{-i}^{1} \mid s_{i}, a\right), \ldots, \pi_{i}\left(s_{-i}^{\left|S_{-i}\right|} \mid s_{i}, a\right)\right)$ is denoted by $\pi_{i}\left(s_{i}, a\right)$. Let $X$ be the set of possible allocations, which is

\footnotetext{
${ }^{9}$ This assumption is introduced to avoid off the equilibrium path where only a strict subset of the agents participate in the mechanism. None of the results is affected by this assumption.

${ }^{10}$ Formally, this is a multistage game with communication (Myerson [31]).
} 
independent of $a$ or $s$.

Throughout the paper, I say a certain property is generic if it is satisfied in an open and full measure subset in $\Xi$. This notion of genericity is stronger than a weaker notion of genericity that only requires the property to hold in an open and dense subset of $\Xi$.

Agent $i^{\prime}$ s payoff $U_{i}-t_{i}-c_{i}\left(a_{i}\right)$ is agent $i$ 's surplus $U_{i}-c_{i}\left(a_{i}\right)$ minus agent $i$ 's transfer. Agent $i$ 's surplus consists of two components: utility and cost. Several classes of utility functions are considered in this paper. The reason is that, for different classes of utility functions, the necessary and sufficient for FSE for an arbitrary profile of utility functions (and cost functions) is different in principle. The most general class of utility functions depend on final allocation $x \in X$, type profile $s \in S$, and action profile $a \in A .{ }^{11}$ Denote the set of such utility function profiles $\left(U(x, s, a)=\left(U_{1}(x, s, a), \ldots U_{n}(x, s, a)\right)\right)$ by $U^{S A}$. The set of utility functions like $U_{i}\left(x, s, a_{i}\right)$ is denoted by $U^{S a}$. I also use $U^{s A}$ and $U^{s a}$, which are similarly defined. ${ }^{12}$

Agent $i$ must pay $c_{i}\left(a_{i}\right) \geq 0$ when he plays $a_{i}$. I assume that every agent has an option to do nothing, that is, $\forall i, \exists a_{i} \in A_{i}$ such that $c_{i}\left(a_{i}\right)=0 .{ }^{13}$ Let $C$ be the space of profiles of such cost functions.

Mechanism $(M, x, t)$ consists of message spaces $M=\left(M_{1}, \ldots, M_{n}\right)$, allocation function $x: M \rightarrow X$, and monetary transfer $t: M \rightarrow \Re^{n}$, where the $i$ th element $t_{i}(m)$ is agent $i$ 's payment to the principal. While agent $i$ 's rent $U_{i}(x, s, a)-t_{i}$ matters only when he participates in the mechanism, $c_{i}\left(a_{i}\right)$ is independent of agent $i$ 's participation decision. Thus agent $i$ 's final payoff is $-c_{i}\left(a_{i}\right)$ if he didn't participate in the mechanism. The principal's payoff is given by the sum of the resulting profit and transfers: $U_{0}(x, s, a)+\sum_{i=1}^{n} t_{i}$. It is assumed that all agents participate in the mechanism in any (almost) efficient equilibrium. ${ }^{14}$ The notion of equilibrium for this game is Bayesian Nash equilibrium.

My first goal is to understand when the principal can implement the most

\footnotetext{
${ }^{11}$ Note that utilities are independent of the agents' participation decision. Since I assume that every agent must participate in the mechanism to implement any (almost) efficient allocation, this assumption is without loss of generality as long as (almost) efficient equilibria are concerned.

${ }^{12}$ For pure information acquisition model, $s$ is a profile of purely informative signals about payoff-relevant hidden parameter $\theta$. If this is the case, $E\left[u_{i}(x, \theta)\right], i=0, \ldots, n$ woudn't depend on $a$ or $s$ directly. Then the sufficient condition for FSE in this paper is valid, but the necessary condition is not. Finding the exact necessary and sufficient condition for FSE in this case is left for futture research.

${ }^{13}$ Reservation values are normalized to 0 .

${ }^{14}$ This assumption is usually satisfied in many applications (auction etc.) and my results can be easily reformulated even when it is not.
} 
efficient action profile and extract the full surplus from the agents. Thus first I focus on the implementation of pure action profiles. In Section 4, the implementation of almost efficien mixed action profiles is considered.

\subsection{Revelation Principle and FSE implementability}

The above mechanism can be simplified in two respects. First, it is without loss of generality to use a direct mechanism, where each agent directly reports his private information $\left(M_{i}=S_{i} \times A_{i}\right)$. This follows from the standard revelation principle argument. Second, the agents do not need to disclose all private information. It is without loss of generality that the agents disclose only their types (not actions) to implement any pure action profile.

Proposition 1 When implementing any pure action profile, it is without loss of generality to set $M_{i}=S_{i}$ for $i \in N$.

Proof. See the appendix.

Basically this simpler direct mechanism is interpreting any announced action profile as the equilibrium action profile in terms of the direct mechanism with $M_{i}=S_{i} \times A_{i}$. Then the agents have a smaller number of deviations under this simpler direct mechanism with $M_{i}=S_{i}$ compared to the original direct mechanism with $M_{i}=S_{i} \times A_{i}$. Therefore every pure strategy equilibrium in the latter mechanism remains an equilibrium in the former mechanism. ${ }^{15}$

Note that this proposition does not hold when the principal implements a mixed action profile: the case in which a realized action is truly private information. In this case, realized actions need to be disclosed for the sake of efficiency because the efficient allocation depends on realized action profiles. Furthermore, such private information may be useful to create more flexible transfers among the agents. I will come back to this issue in Section 4 when the implementation of mixed action profiles is considered.

Given Proposition 1, the mechanism design problem reduces to a few simple inequality constraints. In the following, I use utility functions in $U^{S A}$ to describe these constraints, but the same constraints apply to the other classes of utility functions. Given any direct mechanism $(S, x, t)$, let $E U_{i}^{x}\left(s_{i}^{\prime}, s_{i}, a\right)$ be agent $i$ 's expected utility when $a$ is played, $s_{i}$ is observed, and $s_{i}^{\prime}$ is announced. That is, $E U_{i}^{x}\left(s_{i}^{\prime}, s_{i}, a\right)=E\left[U_{i}\left(x\left(s_{i}^{\prime}, s_{-i}\right), s, a\right) \mid s_{i}, a\right]$. To implement $x$ and $a^{*}$,

\footnotetext{
${ }^{15}$ I thank Jacques Crémer for this interpretation.
} 
the following constraints need to be satisfied for some $t$ :

$$
\begin{aligned}
\forall a_{i} \in & A_{i}, \sigma_{i}: S_{i} \rightarrow S_{i}, \text { and } i \in N, \\
& \sum_{s_{i} \in S_{i}} \pi_{i}\left(s_{i} \mid a^{*}\right)\left\{E U_{i}^{x}\left(s_{i}, s_{i}, a^{*}\right)-\pi_{i}\left(s_{i}, a^{*}\right) \cdot t_{i}\left(s_{i}\right)\right\}-c_{i}\left(a_{i}^{*}\right) \\
\geq & \sum_{s_{i} \in S_{i}} \pi_{i}\left(s_{i} \mid a_{i}, a_{-i}^{*}\right) \max \left\{\begin{array}{c}
E U_{i}^{x}\left(\sigma_{i}\left(s_{i}\right), s_{i},\left(a_{i}, a_{-i}^{*}\right)\right) \\
-\pi_{i}\left(s_{i},\left(a_{i}, a_{-i}^{*}\right)\right) \cdot t_{i}\left(\sigma_{i}\left(s_{i}\right)\right)
\end{array}, 0\right\}-c_{i}\left(a_{i}\right) .
\end{aligned}
$$

This means that no agent would gain by deviating to any action other than $a_{i}^{*}$ and/or any announcement/participation strategy. In particular, this constraint implies that the following interim IR constraints are satisfied on the equilibrium path,

$$
\forall s_{i} \in S_{i}, \forall i \in N, E U_{i}^{x}\left(s_{i}, s_{i}, a^{*}\right)-\pi_{i}\left(s_{i}, a^{*}\right) \cdot t_{i}\left(s_{i}\right) \geq 0
$$

and the standard truth-telling constraints are satisfied on the equilibrium path,

$$
\begin{aligned}
\forall s_{i}, s_{i}^{\prime} \in & S_{i}, \forall i \in N \\
& E U_{i}^{x}\left(s_{i}, s_{i}, a^{*}\right)-\pi_{i}\left(s_{i}, a^{*}\right) \cdot t_{i}\left(s_{i}\right) \\
\geq & E U_{i}^{x}\left(s_{i}^{\prime}, s_{i}, a^{*}\right)-\pi_{i}\left(s_{i}, a^{*}\right) \cdot t_{i}\left(s_{i}^{\prime}\right) .
\end{aligned}
$$

When every agent participates in the mechanism and $(x, s, a)$ is realized, the social surplus is given by $\sum_{i=0}^{n} U_{i}(x, s, a)-\sum_{i=1}^{n} c_{i}\left(a_{i}\right)$. For any $a \in A$, let $x^{a}(\cdot)$ be the optimal allocation which maximizes the total expected surplus:

$$
E\left[\sum_{i=0}^{n} U_{i}\left(x^{a}(s), s, a\right) \mid a\right]-\sum_{i=1}^{n} c_{i}\left(a_{i}\right)
$$

Assume that the optimal allocation exists and denote the maximized social surplus by $W(a)$. Let $A^{*}(U, c)$ be the set of action profiles that maximize $W(a)$ given $U \in U^{S A}$ and $c=\left(c_{1}, \ldots, c_{n}\right) \in C$. An action profile $a$ is FSEimplementable when $a$ is implementable and the social surplus given $a$ can be fully extracted.

Definition 1 Given $(U, c)$, an action profile $a \in A$ is FSE-implementable if there exists a mechanism $(S, x, t)$ and Bayesian Nash equilibrium where a is played and the full surplus is extracted from the agents, i.e.

$$
\sum_{s \in S} \pi(s \mid a) \sum_{i=1}^{n} t_{i}(s)=W(a)-E\left[U_{0}\left(x^{a}(s), s, a\right) \mid a\right]
$$

Definition 2 An information structure $(\pi, S, A)$ has FSE property for $U^{Z}$ $(Z \in\{S A, S a, s A, s a\})$ if, for any $U \in U^{Z}$ and $c \in C$, there exists $a \in A^{*}(U, c)$ that is FSE implementable. 


\section{The Full Surplus Extraction Theorem with Hidden Actions}

\subsection{Example: Auction with Espionage}

I start with a simple example to illustrate the main result. Consider the following auction with two bidders $i=1,2$. Bidder $i$ 's true value $v_{i}$ is either $\bar{v}$ or $\underline{v}$, $\bar{v}>\underline{v}>0$, which is not observed before the game starts. The joint distribution of $v=\left(v_{1}, v_{2}\right)$ is affiliated: $\operatorname{Pr}\left(v_{1}=\bar{v}, v_{2}=\bar{v}\right)=\operatorname{Pr}\left(v_{1}=\underline{v}, v_{2}=\underline{v}\right)=\frac{\lambda}{2}$ and $\operatorname{Pr}\left(v_{1}=\bar{v}, v_{2}=\underline{v}\right)=\operatorname{Pr}\left(v_{1}=\underline{v}, v_{2}=\bar{v}\right)=\frac{1-\lambda}{2}$ for $\lambda>\frac{1}{2}$. The bidders observe private signals $s_{i} \in S_{i}=\{h, l\}, i=1,2$. They can choose one of two actions, $N$ or $S$ before they play the auction. If bidder $i$ chooses $N$ ("no spying"), then he observes his true value $v_{i}\left(\operatorname{Pr}\left(s_{i}=h \mid v_{i}=\bar{v}\right)=\operatorname{Pr}\left(s_{i}=l \mid v_{i}=\underline{v}\right)=1\right)$. If bidder $i$ chooses $S$ ("spying"), which costs $c>0$ (the cost of $N$ is normalized to 0$)$, then he observes the other bidder's value $v_{j}\left(\operatorname{Pr}\left(s_{i}=h \mid v_{j}=\bar{v}\right)=\right.$ $\left.\operatorname{Pr}\left(s_{i}=l \mid v_{j}=\underline{v}\right)=1\right) .{ }^{16}$ When bidder $i$ chooses $S$, he does not learn his own value. The efficient action profile is always $(N, N)$ because it reveals the bidders' true values with no cost. So the question is whether $(N, N)$ can be implemented and the full surplus can be extracted at the same time.

In the following, it is shown that FSE is impossible when, for example, $\bar{v}$ is large. ${ }^{17}$ The idea is that the principal needs to exploit correlation of the private signals to extract full surplus from the bidders. In particular, a bidder's transfer depends on the other bidder's announcement. Then each bidder has incentive to spy on the other bidder to learn the other bidder's signal.

Note that this is a simple affiliated private value auction given $(N, N)$. If $N$ is the only available action, then this is exactly the case where FSE is obtained according to CM's condition. The point of this example is to show that the presence of another action may keep a principal from extracting full surplus.

Consider a direct mechanism without loss of generality. For simplicity, assume that each bidder wins with equal probability when their announced types are the same. Suppose that the auctioneer is able to extract full surplus from the bidders. Since the low type's interim rent must be 0 in equilibrium,

$$
\frac{\lambda}{2} \underline{v}-\sum_{s_{j}} t_{i}\left(l, s_{j}\right) \pi_{i}\left(s_{j} \mid l, N N\right)=0
$$

\footnotetext{
${ }^{16}$ Fang and Morris [14] analyze two bidder private value auctions in which each bidder observes his own value as well as a noisy signal of the other bidder's value.

${ }^{17} \mathrm{FSE}$ is also impossible when $c>0$ is small enough.
} 
The truth-telling constraint for the high type is

$$
\begin{aligned}
& \left\{\frac{\lambda}{2}+(1-\lambda)\right\} \bar{v}-\sum_{s_{j}} t_{i}\left(h, s_{j}\right) \pi_{i}\left(s_{j} \mid h, N N\right) \\
\geq & \frac{1-\lambda}{2} \bar{v}-\sum_{s_{j}} t_{i}\left(l, s_{j}\right) \pi_{i}\left(s_{j} \mid h, N N\right)
\end{aligned}
$$

Note that left hand side of this truth-telling constraint is the high type's interim rent, thus it must be 0 . Now let $\bar{v}$ go to $\infty$. Then (2) imply that the high type's expected payment $\sum_{s_{j}} t_{i}\left(l, s_{j}\right) \pi\left(s_{j} \mid h, N N\right)$ from lying converges to $\infty$. It is not difficult to see from (1) that this can be only accomplished by letting $t_{i}(l, l) \rightarrow-\infty$ and $t_{i}(l, h) \rightarrow \infty$, that is, by rewarding "positive" correlation and punishing "negative" correlation of announcements. Then, when $\bar{v}$ is large, bidder $i$ has a profitable deviation: play $S$, announce $l$ if $s_{i}$ is $l$ and stay out of the mechanism if $s_{i}$ is $h$. The problem is that a unilateral deviation from $N$ to $S$ makes the bidders' private signals even more correlated than in equilibrium.

Hence the following result is obtained.

Proposition 2 Fix parameters $\underline{v}>0$ and $\lambda>\frac{1}{2}$. Then there exists $\widetilde{v}>\underline{v}$ such that $(N, N)$ is not FSE implementable if $\bar{v} \geq \widetilde{v}$.

\subsection{The Full Surplus Extraction Theorem with Hidden Actions}

If there is no action, or equivalently, the action set is a singleton for every agent, then this problem reduces to the standard full surplus extraction problem studied by CM. So it may be useful to state their theorem in the current framework. Let's use $\pi_{i}\left(s_{i}\right),(\pi, S)$ and $U^{s}$ instead of $\pi_{i}\left(s_{i}, a\right),(\pi, S, A)$ and $U^{S A}$ as there is no action. Denote a convex hull of $m$ vectors $x_{i}, i=1, \ldots, m$ in $\Re^{n}$ by $c o\left\{x_{i}: i=1,2, \ldots, m\right\}$. Then CM's theorem can be stated in the following form.

Theorem 3 (Crémer and McLean [13]) An information structure $(\pi, S)$ has FSE property in $U^{s}$ if and only if $\pi_{i}\left(s_{i}\right) \notin \operatorname{co}\left\{\pi_{i}\left(s_{i}^{\prime}\right): s_{i}^{\prime} \in S_{i} /\left\{s_{i}\right\}\right\}$ for all $s_{i} \in S_{i}$ and $i \in N$.

This full surplus extraction theorem can be generalized to the current setting with hidden actions. It turns out that the following analogue of CM's 
condition is the right extension:

$$
\begin{aligned}
& \pi_{i}\left(s_{i}, a\right) \notin \operatorname{co}\left\{\pi_{i}\left(s_{i}^{\prime},\left(a_{i}^{\prime}, a_{-i}\right)\right):\left(s_{i}^{\prime}, a_{i}^{\prime}\right) \neq\left(s_{i}, a_{i}\right)\right\} \\
& \text { for all } s_{i} \in S_{i}, i \in N \text { and } a \in A
\end{aligned}
$$

Like CM's condition, this generalized CM condition (GCM) means that each conditional distribution is not a positive linear combination of the other conditional distributions. The difference is that the pool of conditional distributions is much larger than before. It takes into account all the conditional distributions given not only realized types but also unilateral deviations in action.

It is easy to verify that GCM is violated in the auction example. Conditional distribution $\pi_{1}\left(s_{1},(N, N)\right)$ is a linear combination of $\pi_{1}(l,(S, N))=$ $(0,1)$ and $\pi_{1}(h,(S, N))=(1,0)$.

Given any $a^{*} \in A$, GCM guarantees the existence of transfer $t$ that satisfy the following condition for any $\left(s_{i}^{\prime}, a_{i}^{\prime}\right) \neq\left(s_{i}, a_{i}^{*}\right)$ :

$$
\pi_{i}\left(s_{i}, a^{*}\right) \cdot t_{i}\left(s_{i}\right)<\pi_{i}\left(s_{i}^{\prime},\left(a_{i}^{\prime}, a_{-i}^{*}\right)\right) \cdot t_{i}\left(s_{i}\right) .
$$

This means that agent $i$ must pay more by playing $a_{i}^{\prime}$ and pretending to be $s_{i}$ given $s_{i}^{\prime}$ than $s_{i}$ would pay in equilibrium. All deviations (with respect to actions and/or announcements) can be deterred by setting $t_{i}\left(s_{i}\right)$ large enough (by multiplying it if necessary). Mathematically, $t_{i}\left(s_{i}\right)$ is a normal vector of a hyperplane that separates $\pi_{i}\left(s_{i}, a^{*}\right)$ from $\left\{\pi_{i}\left(s_{i}^{\prime},\left(a_{i}^{\prime}, a_{-i}^{*}\right)\right):\left(s_{i}^{\prime}, a_{i}^{\prime}\right) \neq\left(s_{i}, a_{i}\right)\right\}$. Condition GCM is equivalent to the existence of such hyperplane for every $a^{*} \in A$.

In the appendix, it is proved that condition GCM is sufficient for FSE with respect to $U^{S A}$. This automatically implies that GCM is sufficient for FSE for $U^{S a}, U^{s A}$ and $U^{s a}$

It can be shown that GCM is also necessary for FSE, but with one caveat. What I need to do is to find a particular type of utility functions for which FSE is not obtained when GCM is violated. To this end, agent $i$ 's utility conditional on $h$ was modified in the auction example. To prove necessity for the general case, I use a similar trick: modify agent $i$ 's utility conditional on some $s_{i}$ or some action $a_{i}$. The problem is that, in general, this may affect the efficient action profile. The auction example is set up so that the efficient action profile is always $(N, N)$. But this property may not be satisfied for the general case. However, when agent $i$ 's utility depends on $a_{i}$, it is possible to modify utility functions while keeping the same efficient action profile. ${ }^{18}$ Thus condition GCM is necessary for FSE for a class of utility functions such as $U^{S A}, U^{S a}, U^{s A}$, and $U^{s a}$.

\footnotetext{
${ }^{18}$ In fact, it suffices to assume that $U_{j}$ depends on $a_{i}$ for some $j \in N \cup\{0\}$ for every $i \in N$.
} 
Combining this necessity result with the sufficiency result, the following theorem is obtained.

Theorem 4 An information structure $(\pi, S, A)$ has FSE property in $U^{S A}$, $U^{S a}, U^{s A}$ and $U^{s a}$ if and only if it satisfies $G C M$.

Proof. See Appendix.

As already mentioned, CM's theorem corresponds to the special case where no action is available to any player.

\subsection{Genericity of FSE Property}

How strong is GCM? Since there are $\left|S_{i}\right| \cdot\left|A_{i}\right|$ different conditional distributions with hidden actions and since each conditional distribution is $\left|S_{-i}\right|$-dimensional vector, the conditional distribution vectors are independent (henceforth satisfy GCM) generically when $\left|S_{i}\right| \cdot\left|A_{i}\right| \leq\left|S_{-i}\right|$. On the other hand, when $\left|S_{i}\right| \cdot\left|A_{i}\right|>\left|S_{-i}\right|$, it is possible to construct a robust example where GCM is violated. This implies that FSE is not obtained generically when there are many actions, i.e. when the agents can manipulate the type distribution in many ways. For example, consider a symmetric model where $\left|S_{i}\right|=m$ and $\left|A_{i}\right|=K$ for all $i$. Then the above weak inequality is satisfied if and only if $K \leq m^{n-2}$. When the number of the agents is two, it is violated as soon as each agent can take two different actions as in the auction example. The following result follows from the proof of Theorem $8 .{ }^{19}$

Theorem 5 FSE property is generic in $\Xi$ if and only if $\left|S_{i}\right| \cdot\left|A_{i}\right| \leq\left|S_{-i}\right|$ for all $i \in N$.

When $\left|A_{i}\right|=1$ (the case of $\mathrm{CM}$ ), this condition reduces to $\left|S_{i}\right| \leq\left|S_{-i}\right|$. Thus it is always satisfied generically for symmetric models. On the other hand, the above result shows that GCM may not be generically satisfied even for symmetric models if the number of actions is much larger than the number of private types.

\footnotetext{
19 "Only if" does not follow from Theorem 8. But, when GCM is violated, it is easy to construct an example where FSE fails.
} 


\subsection{Discussion}

\section{On Assumption of Commitment}

It is important for the above results that a mechanism is proposed before the agents act, that is, the principal commits not to change the mechanism once actions are taken. An alternative assumption would be to allow the principal to propose a mechanism after actions are taken. Which model is more appropriate depends on the nature of problems at hand. For example, the former assumption may be more natural if the principal is uncertain about the timing of actions. It would be also natural if the principal designs a mechanism which will be played repeatedly (repeated auctions etc.) because the strategic complexity of such dynamic games is significantly reduced with commitment. Both assumptions have been employed in the literature. For example, Crémer and Khalil [10], Crémer, Khalil and Rochet[11], and Lewis and Sappington [23] use the former assumption, whereas Crémer, Khalil, and Rochet [12] use the latter assumption.

The previous analysis suggests that a principal has a strong incentive to commit to a mechanism in the beginning of the game. Suppose that the principal cannot commit. Given that a pure action profile is implemented, since the principal knows the action profile in equilibrium before she proposes a mechanism, she can extract all the expected utility (not the surplus) of the agents as long as CM's condition (which is typically generic) is satisfied given the equilibrium action profile. Hence the agents are not compensated for their (sunk) cost. This means that the only cheapst action profile is implementable without commitment. This is not desirable for a principal for two reasons. First, the most efficent action profile may not be implemented without commitment, thus the total size of the pie, from which the principal extracts surplus, may not be maximized. Second, the principal may simply make less money without commitment. In the auction example, the only implementable pure action profile is $(N, N)$ without commitment. Although $(N, N)$ is efficient, the principal may not extract the full surplus. However, it is proved in the next section that $(N, N)$ is approximately FSE implementable (using a mixed strategy profile converging to $(N, N))$ with commitment. ${ }^{20}$ See the next section for details.

\section{Interim and Ex Ante IR Constraint}

Suppose that the agents sign a contract before taking any action and cannot run away without the principal's consent. That is, the agents are subject to

\footnotetext{
${ }^{20}$ It may be possible to implement some mixed action profile without commitment. But it can be shown that even approximate FSE is not obtained in this case.
} 
ex ante IR constraints rather than interim IR constraints. In this case, it is significantly easier for a principal to extract full surplus. Since the principal can use "entrance fee", the necessary and sufficient condition for FSE reduces to a much weaker condition, namely, the necessary and sufficient condition for the efficient (Bayesian) implementation. For example, a condition similar to Assumption 1 (ii) in Aoyagi [2] would suffice for FSE. ${ }^{21}$

\section{Universal Type Space}

Some papers study FSE in more general type spaces. Neeman [32] shows that FSE fails when some agent can be two different types with different values who share the same belief about the other agents' types. This type of information structure is not generic when each agent's type space is a finite set of payoff-relevant types. However, if one consider universal type spaces (à la Mertens and Zamir [29]), the "naive" type space that is generated from such space with a common prior is very special. Thus FSE may be "more likely" to fail in the space of more general type spaces. In fact Heifetz and Neeman [18] show that the set of priors on a universal type space which allows FSE is "small" in a certain sense. ${ }^{22}$ My results are complementary to theirs because they show that FSE can fail even in a naive type space.

\section{FSE and Information Acquisition}

Parreiras [35] is closely related to this paper. In his model, each agent draws two types of private information $\left(t_{i}, \theta_{i}\right) \in T_{i} \times \Theta_{i}$, where $t_{i}$ is a payoff relevant type and $\theta_{i}$ is a parameter which reflects the informativeness of $t_{i}$ about $t_{-i}$. He shows that FSE property does not hold if there are two types $\theta_{i}^{\prime}$ and $\theta_{i}^{\prime \prime}$ such that $i$ 's conditional distribution on $T_{-i}$ given $\left(t_{i}, \theta_{i}^{\prime}\right)$ is more informative than $i$ 's conditional distribution on $T_{-i}$ given $\left(t_{i}, \theta_{i}^{\prime \prime}\right)$ in the sense of Blackwell. This information parameter $\theta_{i}$ is playing the same role as actions in this paper. This paper extends Parreira's analysis by endogenizing $\theta_{i}$ as a degree of information acquisition and proving that FSE may not be a generic property.

\footnotetext{
${ }^{21}$ His condition needs to be modified to allow conditional distributions that depend on actions. But otherwise it is straightforward to apply his result here.

${ }^{22}$ For another attempt to introduce more general type spaces to mechanism design problems, see Bergemann and Morris [5].
} 


\section{Curse of Full Surplus Extraction Theorem}

In this section, FSE is replaced with a weaker notion: approximate FSE. Since mixed action profiles are used to establish approximate FSE, Proposition 1 does not apply. Thus I use the direct mechanism where the agents report both realized actions and their types. The notion of FSE-implementability can be extended to mixed action profiles as follows.

Definition 3 Given $(U, C)$, a mixed action profile $\alpha \in \prod_{i=1}^{n} \triangle\left(A_{i}\right)$ is FSEimplementable if there exists a mechanism $(S \times A, x, t)$ and a perfect Bayesian Nash equilibrium where $\alpha$ is played and the full surplus is extracted from the agents for each realized action profile, i.e.,

$$
\sum_{s \in S} \pi(s \mid a) \sum_{i=1}^{n} t_{i}(s, a)=W(a)-E\left[U_{0}\left(x^{a}(s, a), s, a\right) \mid a\right] \text { for any } a \in \text { supp } \alpha
$$

where suppo is the support of $\alpha \in \prod_{i=1}^{n} \triangle A_{i}$.

Definition 4 An information structure $(\pi, S, A)$ has approximate FSE property for $U^{Z}\left(Z=S A, S a\right.$ etc.) if, for any $\varepsilon>0, U \in U^{Z}$ and $c \in C$, there exists $\alpha \in \prod_{i=1}^{n} \triangle\left(A_{i}\right)$ which is FSE implementable and satisfies

$$
\left|W\left(a^{*}\right)-E\left[U_{0}\left(x^{a}(s, a), s, a\right) \mid \alpha\right]-E\left[\sum_{i=1}^{n} t_{i}(s, a) \mid \alpha\right]\right|<\varepsilon
$$

for some $a^{*} \in A^{*}(U, c)$ for some mechanism $(S \times A, x, t)$.

Let's call a realized type-action pair $\left(s_{i}, a_{i}\right)$ player $i$ 's extended type. The crucial insight is that a set of conditional distributions in the space of extended types satisfies CM's necessary and sufficient condition much more easily given any completely mixed action. Thus every almost efficient mixed action profile can be FSE-implementable even when the most efficient action is not FSEimplementable. When CM's condition holds in the extended type space given any completely mixed action profile, the principal can extract the full surplus from each extended type of every agent. In particular, any cost associated with actions can be exactly reimbursed by the mechanism at the end of the game. This in turn provides the incentive for the agents to randomize in the beginning of the game. 


\subsection{Approximate FSE}

To understand how useful the extended type space is, the following simple example might be useful. Suppose that there are two agents with $S_{1}=$ $\left\{x_{1}, x_{2}, x_{3}\right\}$ and $S_{2}=\left\{y_{1}, y_{2}\right\}$. There is no action for agent 1 , and $A_{2}=$ $\left\{a^{\prime}, a^{\prime \prime}\right\}$. Suppose that agent 1's conditional belief on agent 2's extended type space $S_{2} \times A_{2}$ is given by the following matrix.

\begin{tabular}{|l|l|l|l|l|}
\hline & $\left(y_{1}, a^{\prime}\right)$ & $\left(y_{2}, a^{\prime}\right)$ & $\left(y_{1}, a^{\prime \prime}\right)$ & $\left(y_{2}, a^{\prime \prime}\right)$ \\
\hline$x_{1}$ & $\alpha \cdot \frac{3}{4}$ & $\alpha \cdot \frac{1}{4}$ & $(1-\alpha)\left(\frac{3}{4}+\varepsilon\right)$ & $(1-\alpha)\left(\frac{1}{4}-\varepsilon\right)$ \\
\hline$x_{2}$ & $\alpha \cdot \frac{1}{2}$ & $\alpha \cdot \frac{1}{2}$ & $(1-\alpha)\left(\frac{1}{2}+\varepsilon^{\prime}\right)$ & $(1-\alpha)\left(\frac{1}{2}-\varepsilon^{\prime}\right)$ \\
\hline$x_{3}$ & $\alpha \cdot \frac{1}{4}$ & $\alpha \cdot \frac{3}{4}$ & $(1-\alpha)\left(\frac{1}{4}+\varepsilon^{\prime \prime}\right)$ & $(1-\alpha)\left(\frac{3}{4}-\varepsilon^{\prime \prime}\right)$ \\
\hline
\end{tabular}

where $\alpha$ is the probability that agent 2 plays $a^{\prime}$.

In this example, if agent 2 plays $a^{\prime}$ with probability $1(\alpha=1)$, then the second row becomes a linear combination of the other two. Thus CM's condition is violated. Similarly, if agent 2 plays $a^{\prime \prime}$ with probability $1(\alpha=0)$, the second row is still a positive linear combination of the first and the third. Notice, however, that the weight for each row is slightly different across these two linear combinations because of $\varepsilon, \varepsilon^{\prime}, \varepsilon^{\prime \prime}>0$. This implies that the second row is not a linear combination of others if agent 2 uses any genuine mixed action profile $(\alpha \in(0,1))$. Therefore this conditional distribution matrix satisfies CM's necessary and sufficient condition (for agent 1 ) if and only if agent 2 mixes his actions. ${ }^{23}$

Let's define CM's condition on the extend type space more formally. For any given completely mixed strategy $\alpha_{-i}$ by other players than agent $i$, let $\pi_{i}\left(s_{i}, a_{i}, \alpha_{-i}\right) \in \Re^{\left|S_{-i} \times A_{-i}\right|}$ be agent $i$ 's belief about the other agents' extended types $\left(s_{-i}, a_{-i}\right) \in S_{-i} \times A_{-i}$ conditional on agent $i$ 's extended type $\left(s_{i}, a_{i}\right) \in$ $S_{i} \times A_{i}$.

Condition 1 (ECM) Given a completely mixed action profile $\alpha \in \prod_{i \in N} \triangle^{o}\left(A_{i}\right)$, an information structure $(\pi, S, A)$ satisfies Extended Crémer-McLean condition (ECM) if

$$
\pi_{i}\left(s_{i}, a_{i}, \alpha_{-i}\right) \notin \operatorname{co}\left\{\pi_{i}\left(s_{i}^{\prime}, a_{i}^{\prime}, \alpha_{-i}\right),\left(s_{i}^{\prime}, a_{i}^{\prime}\right) \neq\left(s_{i}, a_{i}\right)\right\}
$$

for any $i \in N$ and any $\left(s_{i}, a_{i}\right) \in S_{i} \times A_{i}$

The above example motivates the following Modified Crémer-McLean condition (MCM).

\footnotetext{
${ }^{23}$ Indeed this matrix is full row rank for almost all $\varepsilon, \varepsilon^{\prime}, \varepsilon^{\prime \prime}$, which is a stronger condition than CM's condition.
} 
Condition 2 (MCM) For any $i \in N$ and any $\left(s_{i}, a_{i}\right) \in S_{i} \times A_{i}$, there exists $a_{-i} \in A_{-i}$ such that

$$
\pi_{i}\left(s_{i}, a\right) \notin \operatorname{co}\left\{\pi_{i}\left(s_{i}^{\prime}, a_{i}^{\prime}, a_{-i}\right):\left(s_{i}^{\prime}, a_{i}^{\prime}\right) \neq\left(s_{i}, a_{i}\right)\right\}
$$

It is not difficult to show that MCM implies ECM for any completely mixed strategy $\alpha$. Being independent of the choice of $\alpha$, MCM is easier to check than ECM. Note that MCM reduces to GCM if $a$ is fixed. Since $a_{-i}$ can be varied for each $a_{i}$, MCM is weaker than GCM.

The basic idea behind these conditions is that, since a combination of actions and types has more information than just types, it is useful to condition continuation strategies on realized actions. This idea is closely related to private strategy (Kandori and Obara [20], Mailath, Matthews, and Sekiguchi [24], Obara [33]), although private strategies in these papers do not use messages. This type of strategy (with messages) is similar to the strategy which is used by Kandori [19] to relax Fudenberg, Levine and Maskin [15]'s sufficient condition for the folk theorem with imperfect public monitoring. However, Kandori [19] examines how the rank condition of conditional distributions can be relaxed by using mixed actions and communication, whereas the above conditions focuses more on how the convex independence condition of conditional distributions can be relaxed.

The following theorems are the main results of this section.

Theorem 6 Given $(U, c)$, a completely mixed action profile $\alpha \in \prod_{i \in N} \triangle^{o}\left(A_{i}\right)$ is FSE implementable if $(\pi, S, A)$ satisfies ECM given $\alpha$.

Theorem 7 An information structure $(\pi, S, A)$ has an approximate FSE property in $U^{S A}, U^{s A}, U^{S a}$, and $U^{\text {sa }}$ if it satisfies $M C M$.

The proof of the first theorem is similar to the proof of Theorem 4, thus omitted. The second theorem follows from the fact that MCM implies ECM for any $\alpha$ and any efficient action profile $a^{*} \in A$ can be approximated by completely mixed action profile.

These results suggest that the presence of hidden actions can be beneficial to the principal contrary to the result in Section 3. For example, suppose that the joint type distribution is independent across agents given the efficient action profile. Then CM's condition is violated and the surplus cannot be fully extracted. Theorem 7 implies that, even in such a case, the principal may be able to extract almost all the surplus by letting the agents play some inefficient actions with small probability.

As expected, approximate FSE is much easier to obtain as the following theorem shows. 
Theorem 8 Approximate FSE property is generic in $\Xi$ if $\left|S_{i}\right|\left|A_{i}\right| \leq\left|S_{-i}\right|\left|A_{-i}\right|$ for all $i \in N$.

Proof. See Appendix.

Let me point out that this condition $\left(\left|A_{i}\right|\left|S_{i}\right| \leq\left|A_{-i}\right|\left|S_{-i}\right|\right)$ is a sufficient condition for the set of conditional distributions for agent $i\left(\pi_{i}\left(s_{-i}, a_{-i} \mid s_{i}, a_{i}, \alpha_{-i}\right)\right.$, $\left.\left(s_{i}, a_{i}\right) \in S_{i} \times A_{i}\right)$ to be full-rank given any completely mixed action profile. ${ }^{24}$ This is much stronger than ECM being satisfied for any completely mixed $\alpha$. I conjecture that ECM is satisfied practically almost always for almost all $\alpha$. Nonetheless, the condition for Theorem 8 is still very weak compared to the condition for Theorem 5. In particular, Theorem 8 implies that the approximate FSE property is generically satisfied for the symmetric case $\left(\left|A_{i}\right|=\left|A_{j}\right|\right.$, $\left|S_{i}\right|=\left|S_{j}\right|$ for all $\left.i, j \in N\right)$ unlike the FSE property.

\subsection{Auction with Espionage Continued}

Let's go back to the auction example. Suppose that player $i, i=1,2$ plays $N$ with probability $\alpha_{i} \in(0,1)$ and $S$ with probability $1-\alpha_{i}$. Then, bidder $i^{\prime}$ s conditional distributions on bidder $j(\neq i)$ 's extended type space $S_{j} \times A_{j}$ is as follows.

\begin{tabular}{|l|l|l|l|l|}
\hline & $(N, h)$ & $(N, l)$ & $(S, h)$ & $(S, l)$ \\
\hline$(N, h)$ & $\alpha_{j} \lambda$ & $\alpha_{j}(1-\lambda)$ & $1-\alpha_{j}$ & 0 \\
\hline$(N, l)$ & $\alpha_{j}(1-\lambda)$ & $\alpha_{j} \lambda$ & 0 & $1-\alpha_{j}$ \\
\hline$(S, h)$ & $\alpha_{j}$ & 0 & $\left(1-\alpha_{j}\right) \lambda$ & $\left(1-\alpha_{j}\right)(1-\lambda)$ \\
\hline$(S, l)$ & 0 & $\alpha_{j}$ & $\left(1-\alpha_{j}\right)(1-\lambda)$ & $\left(1-\alpha_{j}\right) \lambda$ \\
\hline
\end{tabular}

This conditional distribution matrix satisfies MCM. Thus it is possible to find some monetary transfers which extract all the surplus from the bidders. This in turn guarantees that they have incentive to randomize.

To be more concrete, let me describe one example of mechanisms that extract full surplus for each realization of action profile. The bidders announce both their types and realized actions. The good is allocated efficiently and symmetrically. There will be transfers if and only if the bidders announce different actions, i.e. when one bidder claims that he spied on the other and the other claims that he did not. Note that this is exactly when private types are perfectly correlated. This strong correlation can be exploited to provide a

\footnotetext{
${ }^{24}$ The full rank condition does not imply MCM, but guarantees ECM for any completely mixed action profile like MCM does.
} 
proper incentive to both players. If a bidder deviates unilaterally to announce a false action or a false type, then his announcement may be inconsistent with the other bidder's announcement. If these inconsistencies are severely punished, then no bidder would have incentive to deviate at the communication stage. Finally transfers can be constructed so that the every bidder's utility is exactly extracted, whereas the cost of spying is reimbursed to the bidder who spied.

\section{Appendix: Proof of the Theorem}

\section{Proof of Proposition 1}

Proof. This proposition is proved for $U^{S A}$ because it is the most general class of utility functions.

The proof consists of two steps. First, clearly it is without loss of generality to use a direct mechanism i.e. $M_{i}=S_{i} \times A_{i}$ by the revelation principle. Next, take any such direct mechanism $(S \times A, x, t)$ and any Bayesian Nash equilibrium. Let $a^{*} \in A$ be the equilibrium action profile. Consider the alternative mechanism $\left(S, x^{\prime}, t^{\prime},\right)$ defined by $x^{\prime}(s)=x\left(s, a^{*}\right)$ and $t^{\prime}(s)=t\left(s, a^{*}\right)$. Then playing $a^{*}$ and revealing true private signals is still an equilibrium of this simpler mechanism, because, for any $a_{i} \in A_{i}, \sigma_{i}: S_{i} \rightarrow S_{i}$ and $i \in N$,

$$
\begin{aligned}
& \sum_{s \in S} \pi\left(s \mid a^{*}\right) E\left[U_{i}\left(x^{\prime}(s), s, a^{*}\right)-t_{i}^{\prime}(s) \mid a^{*}\right]-c_{i}\left(a_{i}^{*}\right) \\
= & \sum_{s \in S} \pi\left(s \mid a^{*}\right) E\left[U_{i}\left(x\left(s, a^{*}\right), s, a^{*}\right)-t_{i}\left(s, a^{*}\right) \mid a^{*}\right]-c_{i}\left(a_{i}^{*}\right) \\
\geq & \sum_{s_{i} \in S_{i}} \pi_{i}\left(s_{i} \mid a_{i}, a_{-i}^{*}\right) \\
& \max \left\{E\left[\begin{array}{c}
\left.\left.U_{i}\left(x\left(\left(\sigma_{i}\left(s_{i}\right), s_{-i}\right), a^{*}\right), s,\left(a_{i}, a_{-i}^{*}\right)\right) \mid s_{i},\left(a_{i}, a_{-i}^{*}\right)\right], 0\right\} \\
-t_{i}\left(\left(\sigma_{i}\left(s_{i}\right), s_{-i}\right), a^{*}\right)
\end{array}\right]\right. \\
= & \sum_{i}\left(a_{i}\right)(\text { by the original equilibrium condition }) \\
& s_{i} \in S_{i}\left(s_{i} \mid a_{i}, a_{-i}^{*}\right) \\
& \left.\max \left\{E\left[\begin{array}{c}
U_{i}\left(x_{i}^{\prime}\left(\sigma_{i}\left(s_{i}\right), s_{-i}\right), s,\left(a_{i}, a_{-i}^{*}\right)\right) \\
-t_{i}^{\prime}\left(\sigma_{i}\left(s_{i}\right), s_{-i}\right)
\end{array}\right)\left(a_{i}, a_{-i}^{*}\right)\right], 0\right\} \\
& -c_{i}\left(a_{i}\right)
\end{aligned}
$$

\section{Proof of Theorem 4}


Proof. (Sufficiency): This is proved for $U^{S A}$. Let $a^{*}$ be any optimal action profile and $x^{*}$ be an optimal allocation. If Condition GCM is satisfied, then there exists $t_{i}^{\prime}: S \rightarrow \Re^{n}$ to satisfy $0=\pi_{i}\left(s_{i}, a^{*}\right) \cdot t_{i}^{\prime}\left(s_{i}\right)<\pi_{i}\left(s_{i}^{\prime},\left(a_{i}^{\prime}, a_{-i}^{*}\right)\right)$. $t_{i}^{\prime}\left(s_{i}\right)$ for every $s_{i},\left(s_{i}^{\prime}, a_{i}^{\prime}\right) \neq\left(s_{i}, a_{i}^{*}\right)$ and $i \in N$ by the hyperplane theorem. Then define $t: S \rightarrow \Re^{n}$ as follows:

$$
t_{i}(s)=\left\{E U_{i}^{x^{*}}\left(s_{i}, s_{i}, a^{*}\right)-c_{i}\left(a_{i}^{*}\right)\right\}+\gamma t_{i}^{\prime}(s),
$$

where $\gamma$ is some large number. Then it is clear that agent $i$ 's surplus is fully extracted by construction if $a^{*}$ is played and the true types are revealed.

Several conditions need to be checked: (1) agent $i$ plays $a_{i}^{*}$, (2) participates in the mechanism on the equilibrium path and (3) reveals his true type on the equilibrium path. (3) is satisfied if $\gamma$ is large enough. (2) is clearly satisfied by construction. To see that (1) is satisfied, note that agent $i$ 's best response after a deviation to any other action is not to participate in the mechanism. Thus agent $i$ 's rent is at most 0 after any such deviation. This proves that $a^{*}$ can be implemented while the principal extracts the full surplus.

(Necessity): This is proved for $U^{s a}$. Suppose that Condition CM is violated for some $s_{i}^{*}$ and $a^{*}$. Then there exists $\left(s_{i, k}, a_{i, k}\right)\left(\neq\left(s_{i}^{*}, a_{i}^{*}\right)\right)$ and $\alpha_{k}>0$, $k=1, \ldots, K$ such that $\pi_{i}\left(s_{i}^{*}, a^{*}\right)=\sum_{k=1}^{K} \alpha_{k} \pi_{i}\left(s_{i, k},\left(a_{i, k}, a_{-i}^{*}\right)\right)$. I derive a contradiction by assuming that the principal can extract full surplus for any $(U, c)$.

Let $\left(U^{\prime}, c\right)$ be any profile of utility functions and cost functions such that (1) $a^{*} \in A^{*}\left(U^{\prime}, c^{\prime}\right),(2) c_{j}\left(a_{j}^{*}\right)=0$ for $i \in N$, and (3) $U_{j}^{\prime}\left(x, s_{j}, a_{j}\right)=0$ for $j \neq i$ and $U_{i}^{\prime}\left(x, s_{i}, a_{i}\right)>0$ for all $\left(x, s_{i}, a_{i}\right)$. By (3), the most efficient allocation is simply the one that maximizes agent $i$ 's utility. Now modify agent $i$ 's utility function as follows

$$
\begin{aligned}
U_{i}\left(x, s_{i}^{1}, a_{i}^{1}\right)= & U_{i}^{\prime}\left(x, s_{i}^{1}, a_{i}^{1}\right)+\eta^{\prime} \text { for }\left(s_{i}^{1}, a_{i}^{1}\right) \\
U_{i}\left(x, s_{i}^{\prime}, a_{i}^{*}\right)= & U_{i}^{\prime}\left(x, s_{i}^{\prime}, a_{i}^{*}\right)+\eta^{\prime \prime} \text { for some } s_{i}^{\prime} \neq s_{i}^{*} \text { if } a_{i}^{1} \neq a_{i}^{*} \\
& \text { and } \\
U_{i}\left(x, s_{i}, a_{i}\right)= & U_{i}^{\prime}\left(x, s_{i}, a_{i}\right) \text { otherwise. }
\end{aligned}
$$

where $\eta^{\prime}, \eta^{\prime \prime}>0$. For each $\eta^{\prime}>0, \eta^{\prime \prime}$ needs to be taken large enough so that $a^{*}$ is still the optimal action profile. Note also that this change of utility functions does not affect the optimal allocation $x^{a^{*}}$.

Let $\left(S, x^{*}, t^{*}\right)$ be an optimal direct mechanism which implements $a^{*}$ and extracts the full surplus with these new utility functions. Consider the following unilateral deviation by agent $i$ for $k=1, \ldots, K$ : play $a_{i, k}$, announce 
$s_{i}^{*}$ when $s_{i, k}$ is observed and stay out otherwise. Agent $i^{\prime}$ s payoff from such a deviation must be less than or equal to agent $i$ 's equilibrium payoff 0 . Thus the following conditions are satisfied for $k=1, \ldots, K$ :

$$
\begin{aligned}
& E U_{i}^{x^{*}}\left(s_{i}^{*}, s_{i}^{*}, a^{*}\right)-\pi_{i}\left(s_{i}^{*}, a^{*}\right) \cdot t_{i}^{*}\left(s_{i}^{*}\right) \\
= & 0 \text { (by FSE }) \\
\geq & \pi_{i}\left(s_{i, k} \mid a_{i, k}, a_{-i}^{*}\right)\left\{E U_{i}^{x^{*}}\left(s_{i}^{*}, s_{i, k},\left(a_{i, k}, a_{-i}^{*}\right)\right)-\pi_{i}\left(s_{i, k},\left(a_{i, k}, a_{-i}^{*}\right)\right) \cdot t_{i}^{*}\left(s_{i}^{*}\right)\right\} \\
& -c_{i}\left(a_{i, k}\right)(\text { by IC) } \\
\geq & E U_{i}^{x^{*}}\left(s_{i}^{*}, s_{i, k},\left(a_{i, k}, a_{-i}^{*}\right)\right)-\pi_{i}\left(s_{i, k},\left(a_{i, k}, a_{-i}^{*}\right)\right) \cdot t_{i}^{*}\left(s_{i}^{*}\right) \\
& -\frac{c_{i}\left(a_{i, k}\right)}{\pi_{i}\left(s_{i, k} \mid a_{i, k}, a_{-i}^{*}\right)}
\end{aligned}
$$

By multiplying (5) by $\alpha_{k}$ and adding up with respect to $k$, I obtain

$$
0 \geq \sum_{k=1}^{K} \alpha_{k}\left(\begin{array}{c}
E U_{i}^{x^{*}}\left(s_{i}^{*}, s_{i, k},\left(a_{i, k}, a_{-i}^{*}\right)\right)-\pi_{i}\left(s_{i, k},\left(a_{i, k}, a_{-i}^{*}\right)\right) \cdot t_{i}^{*}\left(s_{i}^{*}\right) \\
-\frac{c_{i}\left(a_{i, k}\right)}{\pi_{i}\left(s_{i, k} \mid a_{i, k}, a_{-i}^{*}\right)}
\end{array}\right)
$$

Remember that

$$
\begin{aligned}
E U_{i}^{x^{*}}\left(s_{i}^{*}, s_{i},\left(a_{i}, a_{-i}^{*}\right)\right) & =E U_{i}^{\prime x^{*}}\left(s_{i}^{*}, s_{i},\left(a_{i}, a_{-i}^{*}\right)\right) \\
\text { for }\left(s_{i}, a_{i}\right) & \notin\left\{\left(s_{i}^{1}, a_{i}^{1}\right),\left(s_{i}^{\prime}, a_{i}^{*}\right)\right\} \\
E U_{i}^{x^{*}}\left(s_{i}^{*}, s_{i}^{1},\left(a_{i}^{1}, a_{-i}^{*}\right)\right) & =E U_{i}^{\prime x^{*}}\left(s_{i}^{*}, s_{i}^{1},\left(a_{i}^{1}, a_{-i}^{*}\right)\right)+\eta \\
E U_{i}^{x^{*}}\left(s_{i}^{*}, s_{i}^{\prime},\left(a_{i}, a_{-i}^{*}\right)\right) & \geq E U_{i}^{\prime x^{*}}\left(s_{i}^{*}, s_{i}^{\prime},\left(a_{i}, a_{-i}^{*}\right)\right) \\
\pi_{i}\left(s_{i}^{*}, a^{*}\right) & =\sum_{k=1}^{K} \alpha_{k} \pi_{i}\left(s_{i, k},\left(a_{i, k}, a_{-i}^{*}\right)\right) .
\end{aligned}
$$

From (6) and (7), I obtain

$$
\begin{aligned}
0 \geq & \sum_{k=1}^{K} \alpha_{k}\left(E U_{i}^{\prime x^{*}}\left(s_{i}^{*}, s_{i, k},\left(a_{i, k}, a_{-i}^{*}\right)\right)-\frac{c_{i}\left(a_{i, k}\right)}{\pi_{i}\left(s_{i, k} \mid a_{i, k}, a_{-i}^{*}\right)}\right) \\
& -\pi_{i}\left(s_{i}^{*}, a^{*}\right) \cdot t_{i}^{*}\left(s_{i}^{*}\right)+\alpha_{1} \eta^{\prime},
\end{aligned}
$$

Since $E U_{i}^{\prime x^{*}}\left(s_{i}^{*}, s_{i}^{*}, a^{*}\right)=\pi_{i}\left(s_{i}^{*}, a^{*}\right) \cdot t_{i}^{*}\left(s_{i}^{*}\right)$ by assumption, I have

$$
E U_{i}^{\prime x^{*}}\left(s_{i}^{*}, s_{i}^{*}, a^{*}\right) \geq \sum_{k=1}^{K} \alpha_{k}\left(\begin{array}{c}
E U_{i}^{\prime x^{*}}\left(s_{i}^{*}, s_{i, k},\left(a_{i, k}, a_{-i}^{*}\right)\right) \\
-\frac{c_{i}\left(a_{i, k}\right)}{\pi_{i}\left(s_{i, k} \mid a_{i, k}, a_{-i}^{*}\right)}
\end{array}\right)+\alpha_{1} \eta^{\prime} .
$$


Note that this expression is independent of the choice of transfers and must be satisfied for any $\eta^{\prime}>0$. This is a contradiction.

\section{Proof of Theorem 8}

Proof. (sketch) For each $a \in A$, order $s \in S$ and regard $\pi\left(s^{k} \mid a\right), k=$ $1, \ldots,|S|-1$ as free parameters $\left(\pi\left(s^{|S|} \mid a\right)=1-\sum_{k=1}^{|S|} \pi\left(s^{k} \mid a\right)\right)$. Then the space of information structure $\Xi$ is represented as

$$
\left\{\pi\left(s^{k} \mid a\right), k=1, \ldots,|S|-1, a \in A \mid \pi\left(s^{k} \mid a\right) \geq 0 \text { and } \sum_{k=1}^{|S-1|} \pi\left(s^{k} \mid a\right) \leq 1\right\}
$$

Suppose that $\left|S_{i}\right|\left|A_{i}\right| \leq\left|S_{-i}\right|\left|A_{-i}\right|$ and consider a $\left|S_{i}\right|\left|A_{i}\right| \times\left|S_{-i}\right|\left|A_{-i}\right|$ matrix where each $\left(s_{i}, a_{i}\right) \times\left(s_{-i}, a_{-i}\right)$ entry is given by $\pi(s \mid a)$. I show that this matrix is full-row rank for an open full measure subset of $\Xi$. This proves the theorem because the matrix of conditional distributions given any completely mixed action profile is full-row rank (hence ECM is satisfied) if this matrix is full row rank. To see this, pick any completely mixed action profile $\alpha$ and consider $\left|S_{i}\right|\left|A_{i}\right| \times\left|S_{-i}\right|\left|A_{-i}\right|$ matrix where each $\left(s_{i}, a_{i}\right)$ row is given by $\pi_{i}\left(s_{i}, a_{i}, \alpha_{-i}\right)$. If the $\left(s_{i}, a_{i}\right)$ - row is multiplied by $\pi_{i}\left(s_{i} \mid a_{i}, \alpha_{-i}\right)$ and the columns corresponding to $a_{-i}$ is divided by $\alpha_{-i}\left(a_{-i}\right)$, then this matrix becomes the above matrix. Hence the rank of these two matrices are the same for any completely mixed action profile $\alpha$.

The proof is based on an induction argument. It is clear that each $1 \times 1$ submatrix (cell) is full-rank $(\neq 0)$ for an open full measure subset of $\Xi$. It is also clear that entries in any pair of two cells are not the same for an open full measure subset of $\Xi$. Let $\Xi_{1}$ be the subset of $\Xi$ where these two conditions are met. Since a finite intersection of open full measure sets is still open and full measure, $\Xi_{1}$ is open and full measure in $\Xi$. Suppose that $\Xi_{n}$ is a subset of $\Xi$ where (1) every $n \times n$ submatrix is full rank and (2) the determinants of two $n \times n$ submatrices is not the same in absolute value for any pair of two $n \times n$ submatrices that do not share exactly the same set of parameters (this is consistent with the definition of $\Xi_{1}$ because $\left|S_{i}\right| \geq 2, i \in N$ ). Let $\Xi_{n+1}$ be the subset of $\Xi_{n}$, which satisfies (1) and (2) with respect to $(n+1) \times(n+1)$ submatrices. In the following, I show that, if $\Xi_{n}$ is an open and full measure set in $\Xi$, then $\Xi_{n+1}$ is an open and full measure set in $\Xi$. Then, by induction, every $\left|S_{i}\right|\left|A_{i}\right| \times\left|S_{i}\right|\left|A_{i}\right|$ submatrix is full rank, therefore the $\left|S_{i}\right|\left|A_{i}\right| \times\left|S_{-i}\right|\left|A_{-i}\right|$ matrix is full-row rank generically.

Suppose that $\Xi_{n}$ is an open and full measure set in $\Xi$. Fix any $(n+1) \times$ $(n+1)$ submatrix $X^{(n+1)}$. Let $\Xi^{\prime} \subset \Xi$ be the set of parameter vectors for which 
$X^{(n+1)}$ is singular. I show that $\Xi^{\prime}$ is closed and has measure 0. Closedness follows immediately from the continuity of the determinant. Let $\widetilde{\Xi}$ be a compact set in $\Xi_{n}$. Since Lebesgue measure is regular, $\Xi_{n}$ can be approximated by $\widetilde{\Xi}$ from the inside, i.e. the measure of $\Xi_{n} / \Xi$ can be made arbitrarily small. Let $\left|X^{(n+1)}\right|$ be the determinant of $X^{(n+1)}$. Remember that the determinant of an $(n+1) \times(n+1)$ matrix can be expanded with respect to the $h$ th row for any $h$ as follows

$$
\left|X^{(n+1)}\right|=\sum_{j=1}^{n+1}(-1)^{h+j} x_{h j}\left|X_{h j}^{(n)}\right|
$$

where $X_{h j}^{(n)}$ is the $n \times n$ matrix that is obtained by deleting the $h$ th row and the $j$ th column from $X^{(n+1)}$ and $x_{i j}$ is $h j$-entry of $X^{(n+1)}$. A similar expression can be obtained for each column. Pick any parameter vector in $\Xi^{\prime} \cap \widetilde{\Xi}$. Then $\left|X^{(n+1)}\right|=0$ at that vector by definition. I show that a derivative of this determinant with respect to some parameter is nonzero. There are two cases. First, suppose that there is a parameter, call it $x_{h j}$, that appears only once in $X^{(n+1)}$. Take a derivative of $\left|X^{(n+1)}\right|$ with respect to this parameter. Then this derivative is $\left|X_{h j}^{(n)}\right|$, which is not 0 by definition of $\Xi_{n}$ (by (1)). Second, suppose that every parameter appears twice in $X^{(n+1)}$. Then it must be the case that $\pi\left(s^{|S|} \mid a\right)$ is an element of $X^{(n+1)}$. Take a derivative of $\left|X^{(n+1)}\right|$ with respect to any parameter in the same row (or the same column) as $\pi\left(s^{|S|} \mid a\right)$. Then the derivative should look like

$$
\left|X_{h j}^{(n)}\right| \pm\left|X_{k l}^{(n)}\right|
$$

It can be shown that these two $n \times n$ matrices do not share exactly the same set of parameters $\left(\left|S_{i}\right| \geq 2, i \in N\right.$ is used here). Hence it is not 0 by definition of $\Xi_{n}$ (by (2)). In either case, this derivative is not 0 . Then by the implicit function theorem, I can find a neighborhood of the parameter vector in which $\Xi^{\prime}$ is a lower dimensional object. Since $\Xi^{\prime}$ is closed, $\Xi^{\prime} \cap \widetilde{\Xi}$ is compact. By compactness, $\Xi^{\prime} \cap \widetilde{\Xi}$ is then a finite union of such lower-dimensional objects in $\Xi$, thus is a 0 measure set. As $\widetilde{\Xi}$ can be arbitrarily close to a full measure set $\Xi_{n}, \Xi^{\prime}$ is a 0 measure set.

Next fix any two distinct $(n+1) \times(n+1)$ submatrices that do not share the exactly same set of parameters. Let $\Xi^{\prime \prime} \subset \Xi$ be the set of parameter vectors for which these two submatrices has determinants which are the same in their absolute value. As before, I can show that $\Xi^{\prime \prime}$ is closed and has measure 0 (even simpler this time). Closedness is obvious. Pick any parameter vector in $\Xi^{\prime \prime} \cap \widetilde{\Xi}$. Take the derivative of the difference of absolute values with respect 
to the parameter which appears in only one of the two matrices. Then the derivative is just a $( \pm)$ determinant of some $n \times n$ matrix, which is not 0 by definition of $\Xi_{n}$. Then it can be shown that $\Xi^{\prime \prime}$ is closed and has 0 measure in exactly the same way as before.

I can repeat this for every $(n+1) \times(n+1)$ matrix and any pair of two $(n+1) \times(n+1)$ matrices that do not share exactly the same set of parameters. Take a finite union of all $\Xi^{\prime}$ and $\Xi^{\prime \prime}$. This finite union is still closed and measure 0 , thus its complement is an open and full measure set in $\Xi$. Since $\Xi_{n+1}$ is the intersection of this complement and $\Xi_{n}$, it is an open full measure set in $\Xi$.

Finally, I can repeat this procedure with respect to every player. Since the number of players is finite, the $\left|S_{i}\right|\left|A_{i}\right| \times\left|S_{-i}\right|\left|A_{-i}\right|$ matrix is full-row rank for every $i \in N$ in an open and full measure set in $\Xi$.

\section{References}

[1] Abreu, D.and A. Sen, (1991), "Virtual implementation in Nash equilibria," Econometrica, 59:997-1022.

[2] Aoyagi, M., (1998), "Correlated types and Bayesian incentive compatible mechanisms with budget balance," Journal of Economic Theory, 79:142151.

[3] Arozamena, L. and E. Cantillon, (2004), "Investment incentives in procurement auctions," Review of Economic Studies, 71:1-18, 2004.

[4] d'Aspremont, C. and ,L.-A. Gerard-Varet, (1982), "Bayesian incentive compatible beliefs," Journal of Mathematical Economics, 10:83-103.

[5] Bergemann, D. and S. Morris, (2005), "Robust mechanism design," Econometrica, 73:1771-1813.

[6] Bergemann, D and J. Valimaki, (2002), "Information acquisition and efficient mechanism design," Econometrica, 70:1007-1033.

[7] Blackwell, D and M.A.Girshick, (1954), "Theory of games and statistical decisions". New York, John Wiley and Sons, Inc.

[8] Cai, H. (2003), "Costly participation and heterogenous preferences in informational committees," mimeo.

[9] Che, Y-K. and J. Kim, (2006), "Robustly collusion-proof implementation," Econometrica, 74:1069-1107. 
[10] Crémer, J. and F. Khalil., (1992), "Gathering Information before signing a contract," American Economic Review, 82:566-578.

[11] Crémer, J and F. Khalil, and J.-C. Rochet, (1998), "Contracts and productive information gathering," Games and Economic Behavior, 25:174193.

[12] Crémer, J and F. Khalil, and J.-C. Rochet, (1998), "Strategic information gathering before a contract is offered," Journal of Economic Theory, 81:163-200.

[13] Crémer, J and R. McLean, (1988), "Full extraction of surplus in Bayesian and dominant strategy auctions," Econometrica, 56:1247-1257.

[14] Fang, H and S. Morris, (2006), "Multidimensional private value auctions," Journal of Economic Theory, 126:1-30.

[15] Fudenberg, D., D.K. Levine and E. Maskin, (1994), "The folk theorem with imperfect public information," Econometrica, 62:997-1040.

[16] Gerardi, D. and L. Yariv, (2005), "Information acquisition in committees", mimeo.

[17] Gershkov, A. and B. Szentes, (2005), "Optimal voting schemes with costly information acquisition," mimeo.

[18] Heifetz, A. and Z. Neeman, (2006), "A generic (im)possibility of full surplus extraction in mechanism design," Econometrica, 74:213-233.

[19] Kandori, M., (2003), "Randomization, communication, and efficiency in repeated games with imperfect public monitoring," Econometrica, 71:345353.

[20] Kandori, M. and I. Obara, (2006), "Efficiency in repeated games revisited: the role of private strategies," Econometrica, 74:499-519.

[21] Kosenok, G. and S. Severinov, (2007), "Individually rational, budgetbalancing Bayesian mechanisms, and the informed principal problem," forthcoming in Journal of Economic theory.

[22] Laffont, J.J. and D. Martimort, (2000), "Mechanism design with collusion and correlation," Econometrica, 68:309-342. 
[23] Lewis, T.R. and D.E.M. Sappington, (1997), "Information management in incentive problems," Journal of Political Economy, 105:796-821.

[24] Mailath, George J., Matthews, Steven A., and Sekiguchi, Tadashi, (2002), "Private strategies in finitely repeated games with imperfect public monitoring," Contributions to Theoretical Economics, Vol. 2 : Iss. 1, Article 2. Available at: http://www.bepress.com/bejte/contributions/vol2/iss1/art2

[25] Martinelli, C., (2006), "Would rational voters acquire costly information?" Journal of Economic Theory, 129:225-251.

[26] Matsushima, H., (1988), "A new approach to the implementation problem," Journal of Economic Theory, 45:128-144.

[27] Matthews, S., (1984), "Information Acquisition in competitive bidding process," In M. Boyer and R. Kihlstrom, editors, Bayesian Models in Economic Theory, pages 181-207. Amsterdam: North-Holland.

[28] McAfee, R.P., and P.J. Reny, (1992), "Correlated information and mechanisms design," Econometrica, 60:395-421.

[29] Mertens, J.F. and S. Zamir, (1985), "Formulation of bayesian analysis for games with incomplete information," International Journal of Game Theory, 10:619-632.

[30] Milgrom, P.R., (1981), "Rational expectations, information acquisition, and competitive bidding," Econometrica, 49:921-943.

[31] Myerson, R.B., (1986), "Multistage games with communication," Econometrica, 54: 323-358.

[32] Neeman, Z., (2004), "The relevance of private information in mechanisms design," Journal of Economic Theory, 117: 55-77.

[33] Obara, I., (2001), "Private information in repeated games," Ph. D. thesis. University of Pennsylvania.

[34] Parreiras, S. (2005), "Correlated Information, mechanism design and informational rents," Journal of Economic Theory, 123:210-217.

[35] Persico, N., (2000), "Information acquisition in auctions," Econometrica, 68:135-148. 
[36] Persico, N., (2004), "Committee design with endogenous information ", Review of Economic Studies, 71:165-191.

[37] Piccione, M and G. Tan, (1996), "Cost-reducing investment, optimal procurement, and implementation by auction," International Economic Review, 37:663-685.

[38] Robert, J., (1991), "Continuity in auction design," Journal of Economic Theory, 55:169-179.

[39] Tan, G., (1992), "Entry and R\&D in procurement contracting," Journal of Economic Theory, 58:41-60.

[40] Sjöström, T., (1996), "Implementation and information in teams," Economic Design, 1:327-341. 